\title{
Crecimiento en niños con diabetes mellitus insulino-dependiente
}

\author{
Andrea Sepúlveda N.'; Jaime Pérez C. '; \\ Iris Mella $G .^{\prime}$
}

\begin{abstract}
Resumen
Objeivo establecer la talla del nño a! momento del diognóstico de la dioberes nellitus insuilno-dependiente

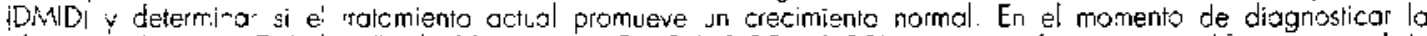
alección, el puntaje $Z$ de la tolla do 81 niños con DMiD $1+0,05 \pm 1.20)$ no era significalivomente díerenle que el de un grupo conircl de 81 niños scnos $|-0,26 \neq 1,04 ; p<0,00|$. De as niños diasét cos, 45 20 hombres y 25 muieres) fueron seguidos durante $6, \varepsilon \pm 3,4$ anos. El puntaje $Z$ de la tailc de los nunos ciaséicos lue menor i $-0,50 \pm$ 1.23 ] comparado con ios controles $1+0.15 \pm 0.77: p<0.03\}$. pero en las niños $\mid-0,91 \pm 0,031$ no se obsenvo dilerencias con el grupo control $(-0.73 \pm 0.63 ; \mathrm{p}=0.53 \mathrm{j}$. El puntaje $Z$ de la talla final a'conzada por los niños diabéticos de ambos sexos fue menor que el de lo talla inicial de e los mismos ip < 0,02 ], sin embargo, er promecio a billa disminuyó moderadamente, no menos de $1 \mathrm{DE}$. Conclusiones: la rolla de las niños con diabeles mellitus dependiente de insulira es normol cuando se hace ei diagnóstico de la entermedad. El tratamiento actual se asocia con una repercusión maderoda de aqué la soore el crecimiento, la que no alca za c csumir significcado pacićgico.
\end{abstract}

(Palabras clave: diabeles mellitus insulino deperidienle, crecimiento, rallo.]

\section{Growth of children with insulin dependent diabetes mellitus}

Objective: to determire if heignt of children of ciagnosis of insu' in dependent diabetes mellitus |DDMI is different than in nomol children, and if theraoy by actual standards pramoles -ormal growlh, records of $8 \mathrm{I}$ diabelic crildren were compared to hose of a same number of normal age rratched controls. Height $Z$ scores ar diagnosis were nol significantly different in diabelic children $;+0.05 \pm 1.20\}$ and controls $\{-0.26 \pm 1.04\} p<0.00$. In 45 diabetic children $\left[20\right.$ boyst which were 'ollowed up tor $6 . B^{\prime} \pm 3.4$ yeors, heigrt shiffed toword smaller $Z$ score volues $1-0.50 \pm$

23) than those of controls $1+0.15 \pm 0.77 ; \mathrm{p}<0.03$ i among boys, but no difierences were recorded amorg diaberic $1-0.73 \pm 0.63 ; p=0.53$ ) and non diabetic girls. Final height $Z$ score of dicbetic children was lower than a diognosis ip $<0.02$ ], however, or the average, this reduction was mild. Conclussion. heichl $Z$ scores of children witr insulin deperdent diabetes mellitus are normal at diagnosis. Growth is afterwards moderately reduced in co ncidence with rearment, but this reduction seems to be withoul pathological inplications.

(Koy words: insulin dependent disoetes mellitus, growh, height.)

El crecimiento de los niños que sufren diabetes mellitus ha sido objeto de numerosas publicaciones y controversias. Entre las primeras descripciones sobre la materia figura el síndrome de Mauriac', caracterizado por trastornos severos del crecimiento, secundarios a aporte in-

1. Departamento de Medicisa. Sección Endocrinología, Hospital Clínico de ta Universidad de Chile. suficiente de insulina, expresados como déficit de talla, obesidad, hepatomegalia y retardo puberal.

Las publicaciones sobre la influencia del control metabólico y la duración de la diabetes en el crecimiento corporal suelen ser contradictorias $^{2-10}$, aunque la mayoría respalda la noción de un efecto deletéreo de la hiperglicemia. También ha sido motivo de discrepancias la estatura en el momento del diagnóstico de la diabetes, 
que para algunos es mayor, mientras para otros es igual o menor que en sujetos normales ${ }^{11,14-16}$.

El crecimiento en niños con diabetes mellitus dependiente de insulina (DMID), fue evaluado en Chile en la década del año $1970^{17}$ cuando no se disponía de autocontrol (glicemia capilar con cintas). La conclusión, en esa época, fue que los niños controlados en un medio hospitalario mostraban déficit manifiesto del peso y la estatura, en especial después de la pubertad. El déficit de la talla era mayor en las mujeres, en quienes la antigüedad de la diabetes tenía mayor impacto que en los varones. En los últimos quince años se han hecho evidentes progresos en el enfoque terapéutico de estos pacientes y se han introducido sistemas sencillos y confiables de autocontrol que pudiesen haber modificado las aludidas tendencias de crecimiento en los niños diabéticos dependientes de insulina.

Esta investigación se realizó con el propósito de evaluar retrospectivamente los efectos de] enfoque terapéutico actual sobre el crecimiento de niños diabéticos dependientes de insulina comparándolo con el de niños no diabéticos. Sus objetivos fueron determinar si la estatura de los niños diabéticos en el momento del diagnostico difiere de la de niños sin diabetes y analizar si la enfermedad repercute sobre la talla alcanzada al término de un determinado período de observación.

\section{Material y Método}

Se estudiaron 81 niños con DMID (42 varones y 39 unujeres, de condición sucioeconomica medio-alra) cuya enfermedad comenzó antes de los 15 arios de edad y habian sido observados, por los autores, un mínimo de dos aho desde el diagnóstico. Ninguno tenfa otra afección crónical al comenzar la diabetes. El Iratamienco recomendado a los pacientes incluyó la alímentación con la dieta habifual para pacientes infantiles diabéticos y distribución individual de los hidratos de carbono según el esquerna de administración de la insulína y la actividad física, Los pacientes se autocontiolaron entre una $y$ tres veces al día. modificando la dosis de insulina de acuerdo a los resultaGos. La insulina se administró en dosis única diaria en $\mathbf{1 5} \%$ de los casos, dos dosis en $5.5 \%$ y múltiples dosis en $30 \%$. La dosis promedio diaria fue de $0,80 \pm 0,20 \mathrm{U} \cdot \mathrm{kg}$ de peso corporal, variando de 0,43 a $l, 50 \mathrm{U} \cdot \mathrm{kg}$. Los médicos tratantes calificaron sólo como regular el contcol logrado sobre la glicemia en la mayoría de los casos.

A partir de la información registrada en las fichas clínicas sobre la edad, peso, talln y duración de la evolución de la diabetes en lós pacientes, se realizó un análisis retros- pectivo, en cortes verticales. El primero en el momento del diagnóstico (inicio) de la diabetes para determinar la talla inicjal en el total de los 81 casos. En el segundo corte, realizado al final del período de observación, sólo fue posible recuperar información de $\mathbf{4 5}$ pacientes (25 mujeres). La edad en el momento de este andilisis y al inicio, así como la duración de la enfenmedad se exponen en la tabla. La estatura de los pacientes y controles se expresó en puntajes $Z$ (Z) o estandarización de las desviaciones escandar según la fortmula:

$$
\mathrm{Z}=\mathrm{x}-\mathrm{X} / \mathrm{SX}
$$

(donde: $x=$ talla individual; $X=$ promedio de la poblacion general; $\mathrm{SX}=$ desviación esiándar de $\mathrm{X}$ ).

La edad en el momento de este análisis y al inicio, asi como la duración de la enfermedad, se exponen en la tabla. La edad de comienzo de las manifestaciones de este grupo fue 5,7 $\pm 3,5$ afros y la duración de la enfermedad basta la evaluación $6,8 \pm 3,4$ años. La escolaridad de los pacientes era normal y la mayoria ( $92 \%$ ) cursaba en el nivel cortespondiente a su edad. Durante el seguimiento $5 \%$ de los pacientes tuvo algún episodio de celoacidosis, 24\% hipoglicemias severas y $10 \%$ infecciones que requirieron hospitalización: 7\% desarrollo retinopatía, 1\% neuropatía. Ninguno mostró signos de nefropatía. En el curso del período de observación. en $3 \%$ se diagnostico una epilepsia, en $9 \%$ enfernedad tiroidea autoimmune y en $4 \%$ vitiligo. Los puntajes $Z$ de las estaturas iniciales de los 81 niños diabéticos incorporados al estudio y las finales de los 45 pacientes que pudieton ser recuperados para la últimb evaluación fueron comparados con iguales números $(n=81$ y 45 respectivamente) de controles constituidos por niños sin antecedentes mórbidos procedentes de colegios de condición socioeconómica similar a la de los pacientes, elegidos al azas y cuya talla fue medida por los autores en el mismo periodo de estudio. La población de referencia se obtuvo del estudio publicado recieniemente por Youlton para es. colares de los mismos niveles econ 6 micos ${ }^{16}$. Se emple $\delta$ la prueba de Student para el cálculo de las diferencias estadísticas en la estatura.

\section{Tabla}

\section{Características de njn̄os con DMID}

\begin{tabular}{lcccc}
\hline Sexo & n & $\begin{array}{c}\text { Edad* } \\
\text { actugl } \\
\overline{\mathbf{x}} \pm \mathbf{D E}\end{array}$ & $\begin{array}{c}\text { Edad* } \\
\text { inicio } \\
\overline{\mathbf{x}} \pm \mathbf{D E}\end{array}$ & $\begin{array}{c}\text { Años } \\
\text { diabetes } \\
\overline{\mathbf{x}} \pm \mathbf{D E}\end{array}$ \\
\hline Hombres & 42 & $17,8 \pm 6,3$ & $7,9 \pm 4,8$ & $9,6 \pm 5,3$ \\
Mujeres & 39 & $16,6 \pm 6,3$ & $7,2 \pm 3,7$ & $9,5 \pm 6,2$ \\
\hline
\end{tabular}

* âtos. 


\section{Resultados}

En los 81 niños inicialmente identificados como diabetes mellitus dependiente de insulina, $\mathrm{Z}$ de la talla inicial era mayor $(+0,05 \pm 1,20)$ que en los controles $(-0,26 \pm 1,04)$, pero las diferencias no alcanzaron significación estadística ( $p=0,06$ ). No se encontraron diferencias entre los niños de uno u otro sexo. La estatura final de los varones diabéticos fue menor
$(Z-0,56 \pm 1,23)$ que en los controles $(Z+0,15 \pm$ 0,$77 ; \mathrm{p}<0,03$ ). En las mujeres las diferencias en la talla final no eran significativas $(Z-0,91$ $\pm 1,45$ ante $Z-0,73 \pm 0,63 ; p=0,53$ ).

$E$ ldeterioro de la estatura se registró tanto en varones como en niñas. El puntaje $Z$ de la talla inicial en varones fue $+0,17 \pm 0,43$ y el de la talla final $-0,56 \pm 1,23(\mathrm{p}<0,02)$. En las mujeres las cifras correspondientes fueron $+0,01 \pm$ 1,21 y $-0,91 \pm 1,45$ ( $p<0,01$ ), como se jlustra en las figuras 1 y 2 .

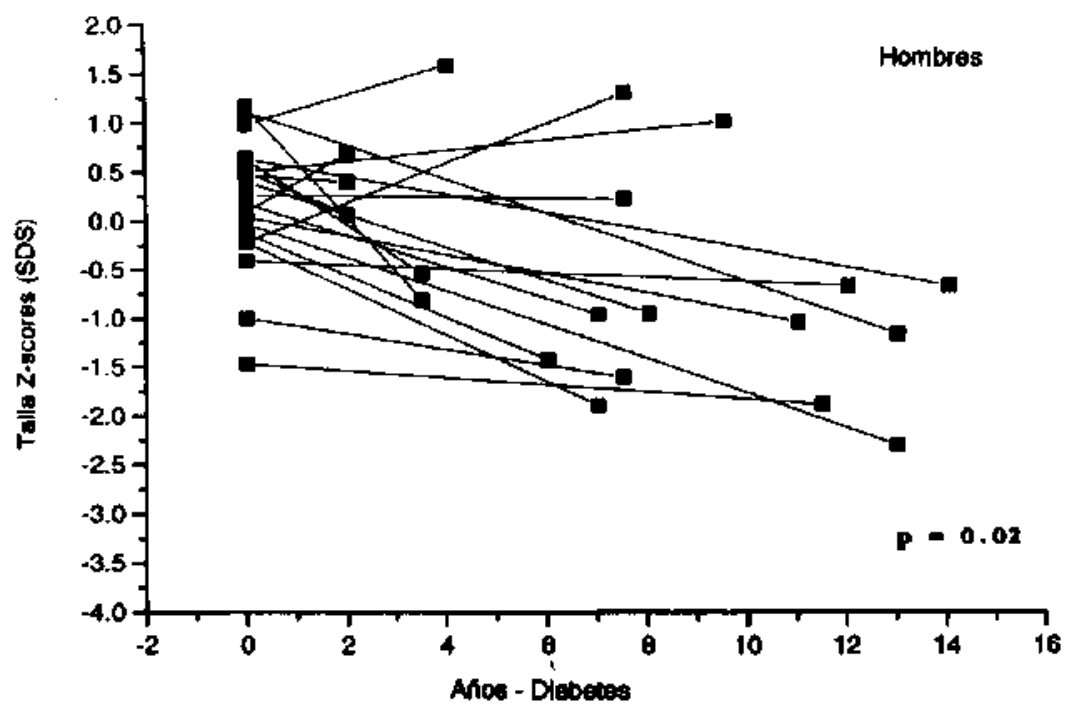

Figura 1: Talla inicial y final, puntajes $Z$ (SUS) de 25 niños con diabetes mellıtus dependienre de insulina $(\bar{x} \pm D E)+0.17 \pm 0,43$ ante $-0.56 \pm 1,23$ (p: 0.02$)$.

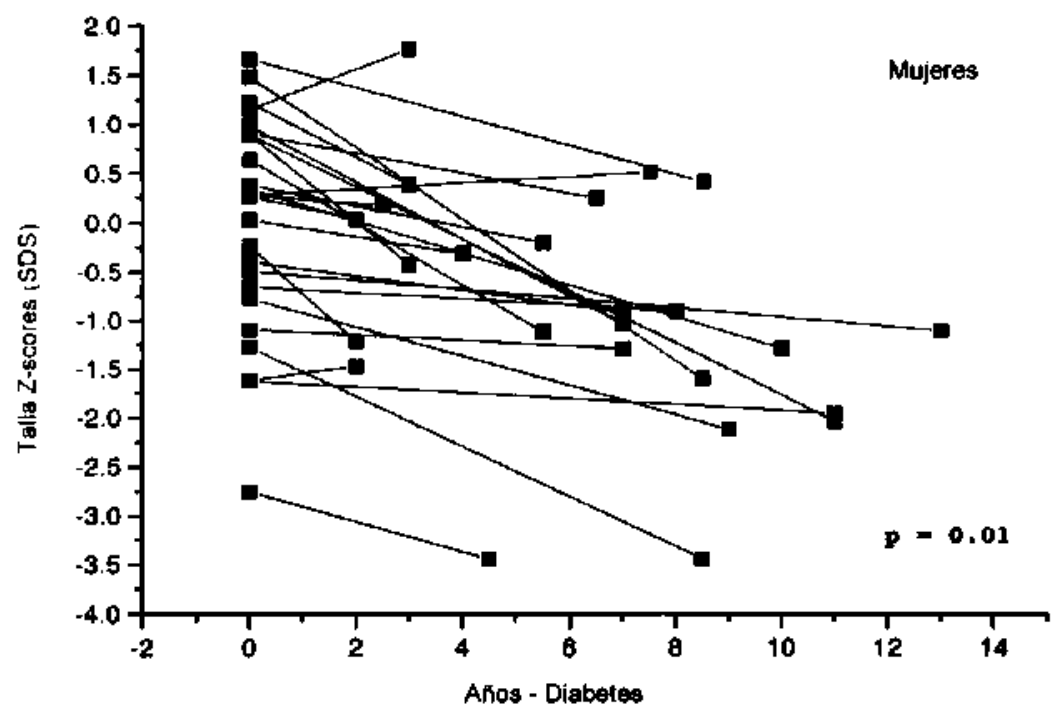

Figura 2: Talla inicial y final, puntaje $Z$ (SDS) de 25 ninas con dabetes mellitus dependiente de insulina $(\bar{x} \pm D E)+0,01 \pm 1,21$ ante $-0,91 \pm 1,45(p=0,01)$. 


\section{Comentario}

La controversia reinante respecto a la talla inicial de los niños con diabetes dependiente de insulina en comparación con niños no diabáticos viene desde largo tiempo. La mayoría de los estudios muesiran que la talla inicial al momento del diagnóstico es mayor que la esperada ${ }^{13}, 15,19-22$. sin embargo, al analizar con detalle cada uno de estos trabajos los resultados son parciales y se refieren a subgrupos clasificados por sexo o edad: en uno solo los varones eran más altos ${ }^{19}$, en otro la diferencia se registraba sólo en niños de 5 a 9 años de edad, no entre los de 10 a 14 años, y los diabéticos de más de 14 años al momento del diagnóstico eran más pequeños que el estándar nacional, mientras la estatura de sus padres y hermanos no diabéticos era comparable a la del patrón empleado ${ }^{15}$. Entre los reportes más recientes. Holl ${ }^{21}$ encontró que la talla era Ievemente superior al estándar no sólo en diabéticos al hacer el diagnóstico, sino tambiên en los hermanos no diabéticos, lo que atribuyen al empleo de estándares basados en información tabulada 20 años antes. Algunos piensan que el aumento de talla precedería al inicio de la diabetes en uno o más años ${ }^{22}$ y que las diferencias se perderian al manifestarse la enfermedad. La mayor estatura en el período preclínico sería un lactor de riesgo, genéticamente determinado, de desarrollar diabeles mellitus infantil. El trabajo de Jjvani ${ }^{5}$, si bien es antiguo y sin grupo control. es interesante porque -empleando como norma las tablas de la misma época (Tanner, 1966)- concluía que la talla inicial de los diabéticos era normal. Hoskins ${ }^{14}$, en gemelos homozigóticos (controles idénticos), encontró que la mitad de los gemelos diabéticos tenía una talla inferior al momento del diagnostico y mientras la otra no mostraba diferencias con sus pares no diabéticos. El mismo grupo de investigadores ${ }^{16}$ siguió en forma prospectiva a los gemelos no diabéticos, encontrando un retardo manifiesto del crecimiento en la fase prediabética de aquellos que posteriormente desarroIlaron la enfermedad. Recientemente en India no se encontró diferencia entre las estaturas de 250 diabéticos, en el momento del diagnóstico, $y$ sus controles ${ }^{23}$. La heterogeneidad de los resultados puede ser explicada por diferencias metodologicas, ausencia de grupo control o falta de vigencia de los estándares en la época del estudio.

Si bien la talla inicial de los diabéticos de nuestra serie fue levemente superior que en los controles, estas diferencias no eran significativas. Estos resultados parecen validados por el empleo de controles y tablas de referencia concordantes con el mismo período de estudio y la situación socioeconómica de los pacientes.

En lo que respecta a la repercusión de la diabetes sobre el desarrollo pondoestatural, desde los primeros trabajos, que sólo empleaban criterios clínicos, se mostraba recuperación en la velocidad de crecimiento cuando mejoraba el control metabólico ${ }^{5 \cdot 7}$.

La optimización del control metabólico mediante tratamiento con bomba de infusión continua o múltiples inyecciones de insulina produce una reducción notoria de la $\mathrm{HbAl}$ y franca mejoría del crecimiento que se asocia a elevación simultánea de la somatomedina C. Del mismo modo, el deterioro de la talla parece secundario al mal control metabólico. El progreso de la estatura al mejorar el control metabólico fue significativo sólo en varones antes de la pubertad. Si la elevación de la $\mathrm{HbAl}$ es moderada (menos de 1,3 veces el límite superior normal) no se afectaría el crecimiento ${ }^{8.9}$. El periodo más vulnerable parece ser el prepuberal ${ }^{10,24}$, cuando hay una importante influencia del metabolismo sobre el crecimiento, pues para que el crecimiento se deteriore una vez establecida la pubertad se requiere que el control de la diabetes sea muy deficiente (HbAl mayor de 16\%). La existencia de clara correlación inversa entre la talla final y la $\mathrm{HbAl}$ corrobora estos hechos ${ }^{25}$. Así pues, la velocidad de crecimiento es fuertemente determinada por el control metabólico pero modulada por la etapa del desarrollo del niño. Si bien el control deficiente es deletéreo, su efecto no es definitivo y se subsana con un manejo metabolico apropiado ${ }^{24 .} 26$.

El mecanismo por el cual el defectuoso control metabólico actúa sobre el crecimiento podría ser un cierto estado de "resístencia" a la hormona de crecimiento, donde la concentración de esta última es alta y la de IGF-1 baja, por aumento de la proteína transportadora o IGF-BPI y de la GH-BP. Lo anterior se debería a falta de respuesta hepática a la $\mathrm{GH}$, semejante a lo que ocurre en la desnutrición severa y al revés que en los obesos ${ }^{27}$. 
De acuerdo con la información presentada, es posible concluir que la estatura de los niños diabéticos al comenzar las manifestaciones de su enfermedad es semejante que la de los no afectados por el trastorno. Si bien el enfoque terapéutico actual no garantiza un crecimiento optimo, en coincidencia con él la talla fínal de los pacientes es satisfactoria, siendo los promedios de las tallas estandarizadas apenas menores que en niños sin diabetes.

\section{Referencias}

I. Thaissonan HS: Mauriax's syndrome. Clin Pediatr 1964: 3: 520-522.

2. Tutersull RB, Pyke DA: Growth in diabetic children: Studies in jdentical twins. Lancet $1973 ; 2: 1105-1) 09$.

3. Thon A, Heinze E, Feiles KD, et al: Development of height and weight in children with diabetes mellitus: report on two prospective multicentre sudies, one cross $\sim$ sectional, one longitudinal. Eur J Pediatr 1992; 1. $\$ 1:$ 258-262

4. Hevber SM. Dunsmore IR: Does control affect growth in diabetes mellitus? Acta Paediatr Scand 1988; 77 : 303-305

5. Sivm 5. Raymer P- Does control influence the growth of diabetic children? Arch Dis Child 1973; 48: 109 I] 5 .

6. Birheck JA: Growth in juvenile diabetes mellitus. Diabetologia 1972; 8: 221-224.

7. Jicksisn RL: Growth and maturation of children with insulin dependent diabetes mellitus. Pedjatr Clin North Am 1984: 3]: 545-567.

8. Rudolf MCJ. Shentin RS, Markowitz R, et al; Effect of intensive insulin treatment on linear growth in the young Jiabetuc patient. J Pediatr 1982; 101: 333-339.

9. Hatder A, Marrero D. Freidenberg G: Impact of glicenic control on growch in children with IDDM. Diabetes 1992; 41 (Suppl 1): 51 A.

10. Wive JE. Kolb EL Sander SE: Effect of glicemic control on growth velocity in children with IDDM. Diabetes Care 1992: 15: 826-839.

11. White P: The child with diabetes In: Marble A, White P. Bradley RF, Krall LP. ed. Joslin's Diabetes Mellitus, Philadelphin. Lea \& Febiger 1971; 339-360.

12. Emmersin AJ, Savige $D C$ : Height at diagrosis in diabetes. Eur J Pediatr 1988; 147; 319-320.
13. Salardi $S$. Tonioli $S$. Tarsoni $P$, et at: Growth and growth factors in diabetes mellitus. Arch Dis Child 1987: 62: 57.62 .

14. Hoskins PJ. Leslie RD, Pyke DA: Height at diagnosis of diabetes in children: a study in identical twins. Brit Med J 1985; 290: 278-280.

15. Sunger TJ. Laporse RE, Tajima $N$, Orchard TI, et al: Height at diagnosis of insulin dependent diaberes in patients and their non-diabetic fonnily members. Brit Med J 1986: 242: 1419-1422.

16. Leslie RD. Lo S, Millward BA, Honour J, Pyke DA: Decreased growth velocity hefore IDDM onset. Diabetologia 1991; 40: 211-216.

17. Mella I, García de los Rlos M. Tapia JC+ et al: Desarolto pondoestatural en diabéticos infantiles. Rev Chil Pediatr 1981; 52: 204-212.

18 Youlsoi $R$. Valenzuela $C$ : Pattón de crecimiento en estatura y peso de 0 a 17 años y de circunsferencia cranearia de 0 a 2 años de nifos de estratos socioeconomicos medio-alto de Santiago. Comparación con el crecimiento de nifios de estratos medio-bajo y bajo del Area Norte de Santíago. Documento de la Rama de Endocrinologia y Genética, Sociedad Chilena de Pediatría (ed), Santiago, 1990.

19. Drayer NM: Height of diahetic children at onset of symploms. Arch Dis Child 1974: 49: 616-620.

20. Price $D E$, Burden $A C$ : Growth of children before onset of diabetes. Diabetes Care 1992; 15: 1393-1395.

2J. Holl RW. Heinze E. Seifert M, Grabert M. Teller WM: Longitudinal analysis of somatic development in paediatric patients with IDDM: genetic influences on height and weight. Diabetologia 1994; 37: 925-929.

22. BIonr L. Persson L. Dahlquist G* A high linear growth is associated with an increased risk of childhood diabetes mellitus. Diabetologia 1992; $35: 528-533$.

23. Ramachandran A, Snehalaha C. Joseph TA, et al: Height at onset of insulia-dependent diabetes in children in southem India. Diabetes Res Clin PTact 1994; 23: 55-5?.

24. Brown $M$, Ahmed $M L$, Clayton $K L$, Dunger $D G$ : Growth during childhood and final height in type I diabeles. Diabetic Med 1994; 2; 82-87.

25. Penfold J, Chase HP. Marshall G, Walravens $\mathrm{CF}$, et af: Final adult height and its relationship to blood glucose control and microvascular complicalions in IDDM. Diabetic Med 1995; 12: 129-133.

26. Lebedev NB: Rates of physical developinent as a marker of the quality of metabolic control in children and adolescents with diabetes mellitus. Probl Endokrinol (Mosk) 1994; 40: 10-12.

27. Holl RW. Siegler B, Scherbaum WA, Heinze E: The serum growth -hormone-binding protein is reduced in young patients with insulın-dependent diabetes mellitus. J Clin Endocrinol Metab 1993; 76: I 65-167. 